\title{
Transport Properties of the Lennard-Jones Truncated and Shifted Fluid from Non-Equilibrium Molecular Dynamics Simulations ${ }^{1}$
}

\author{
Martin P. Lautenschlaeger, Hans Hasse \\ Laboratory of Engineering Thermodynamics (LTD), University of Kaiserslautern, \\ Erwin-Schrödinger-Straße 44, 67663 Kaiserslautern, Germany
}

\begin{abstract}
The thermal conductivity $\lambda$, shear viscosity $\eta$, and self-diffusion coefficient $D$ of the Lennard-Jones fluid truncated and shifted at the cut-off radius $r_{\mathrm{c}}=2.5 \sigma$ (LJTS fluid) are determined for a wide range of thermodynamic states $\left(T^{*}=[0.6,10.0]\right.$ and $\left.\rho^{*}=[0.2,1.2]\right)$. The simulations are carried out using a non-equilibrium molecular dynamics (NEMD) method that was introduced recently and in which two gradients are applied simultaneously. It is shown that the two-gradient method is well-suited for studies of liquid and supercritical states. Data for $\lambda, \eta$, and $D$ for about 350 state points are reported. Two variants of the simulation method, which differ in the accuracy and efficiency, are explored and found to yield consistent data. Correlations for $\lambda, \eta$, and $D \rho$ of the LJTS fluid are provided. The data and the correlations are compared to literature data of Lennard-Jones (LJ) type fluids and good agreement is observed. The truncation of the LJ potential causes a slight increase in $D$, while it has no significant effect on $\lambda$ and $\eta$.

Keywords: NEMD, thermal conductivity, shear viscosity, self-diffusion, correlation
\end{abstract}

\section{Introduction}

The Lennard-Jones (LJ) potential is one of the simplest potentials with which thermophysical properties of simple nonpolar fluids can be described accurately [1-4]. The truncated and shifted LJ potential is computationally particularly efficient. Different cut-off radii $r_{\mathrm{c}}$ are used for the truncation. The whole class of these potentials is called 'LJ-type'

\footnotetext{
${ }^{1}$ The Version of Record of this manuscript is in press for FLUID PHASE EQUILIBRIA 22 Oct 2018 DOI:10.1016/j.fluid.2018.10.019
} 
potentials in the following. LJ-type potentials are often used as model fluids for studying nano-scale processes [5].

Truncating and shifting the Lennard-Jones potential at $r_{\mathrm{c}}=2.5 \sigma$ is common [5, 6]. This fluid is also studied in the present work and in the following simply referred to as 'LJTS fluid'. To the best of our knowledge there is no comprehensive data set on the transport properties thermal conductivity $\lambda$, shear viscosity $\eta$, and self-diffusion coefficient $D$ of the LJTS fluid in the literature. Also for other LJ-type potentials there are only a few studies that cover a wide range of states in a systematic way [7 9]. Thus, there is still a lack of reliable and accurate data of transport properties for LJ-type fluids, especially for $\lambda$.

In the present work $\lambda, \eta$, and $D$ of the LJTS fluid are determined over a wide range of thermodynamic states $\left(T^{*}=[0.6,10.0]\right.$ and $\left.\rho^{*}=[0.2,1.2]\right)$. More than 1000 new data points are provided. The simulations are carried out using the 'two-gradient method' which has been introduced recently [10]. It is characterized by a superposition of a temperature gradient and a velocity gradient, such that $\lambda$ and $\eta$ can be calculated simultaneously. Additionally, $D$ is determined from the mean squared displacement. This approach is feasible, since cross-effects were found to be negligible, even for large gradients [10]. Here, the two-gradient method is applied in two different variants that are referred to as 'global method' and 'local method' following [10]. In the global method, small gradients are used and an average along the gradient is taken to determine the value of the respective transport property for one state point. In the local method, the temperature gradient is large and data for different temperatures is taken based on the assumption of local equilibrium [11-13]. The accuracy of both methods is similar to those of other methods for studying transport properties that have been reported in the literature. The efficiency of the local method is up to one order of magnitude higher than that of the global method. This is particularly remarkable as already the global method, which yields results for three transport properties in one simulation run, is more efficient than common NEMD methods for determining transport properties.

Based on the data determined with the global method empirical correlations for $\lambda$, $\eta$, and $D \rho$ are developed. The correlations are compared with the values obtained from the local method. It is shown that both data sets agree well. This confirms that the 
hypothesis of local equilibrium is valid over a wide range of thermodynamic states, which has already been shown in the literature [14-16]. In addition, the correlations are also compared to data sets from the literature for $\lambda, \eta$, and $D$ of different LJ-type fluids. Thereby, it is shown that for $r_{\mathrm{c}} \geq 2.5 \sigma$ a truncation of the LJ potential has hardly any effect on the transport properties $\lambda$ and $\eta$. This is in accordance with findings of several authors [6, 8, 9, 17 19]. However, we find that a truncation of the LJ potential influences $D$ by increasing the particle mobility.

Altogether, it is shown that the new two-gradient method is robust, highly efficient, and yields accurate results over a wide range of thermodynamic states. It should, however, not be applied for low-density gas-like states.

This paper is organized as follows: in Section 2 the model fluid is defined and the simulation setup is briefly described. In Section 3, an overview of the literature data of transport properties of LJ-type fluids is given. In Section 4 the simulation study is described and the results are presented. The correlation of the data is the topic of Section 5 . In Section 6 the correlations of the different transport properties are compared to results from the present work as well as to the literature data set. Finally, conclusions are drawn in Section 7.

\section{Molecular Model and Simulation Setup}

Molecular model. The LJTS potential used in the present study is

$$
u^{\mathrm{LJTS}}\left(r_{i j}\right)= \begin{cases}u^{\mathrm{LJ}}\left(r_{i j}\right)-u^{\mathrm{LJ}}\left(r_{\mathrm{c}}\right), & r_{i j}<r_{\mathrm{c}} \\ 0, & r_{i j} \geq r_{\mathrm{c}}\end{cases}
$$

where

$$
u^{\mathrm{LJ}}\left(r_{i j}\right)=4 \varepsilon\left[\left(\sigma / r_{i j}\right)^{12}-\left(\sigma / r_{i j}\right)^{6}\right]
$$

$\varepsilon$ is the energy parameter, describing dispersive attraction and $\sigma$ is the size parameter describing the repulsion. $r_{i j}$ is the distance between the two LJTS particles $i$ and $j$. In the present study, the cut-off radius is $r_{\mathrm{c}}=2.5 \sigma$. For brevity, we will generally omit the specification of $r_{\mathrm{c}}$ in the text when we refer to our own data. In the following, all observables are given in reduced units (marked by the asterisk), cf. Table 1. 
Table 1: Definition of the physical quantities in reduced units (marked by the asterisk). The corresponding observable carrying a dimension is plain. $M$ is the mass of a single particle. $k_{\mathrm{B}}$ is the Boltzmann's constant.

\begin{tabular}{lr||lr}
\hline Length & \multicolumn{1}{c}{$L^{*}=\frac{L}{\sigma}$} & Velocity & $\boldsymbol{v}_{k}^{*}=\frac{\boldsymbol{v}_{k}}{\sqrt{\varepsilon / M}}$ \\
Time & $\tau^{*}=\frac{\tau}{\sigma \sqrt{M / \varepsilon}}$ & Shear rate & $\gamma^{*}=\frac{\gamma}{\sqrt{\varepsilon / M} / \sigma}$ \\
Mass & $m^{*}=\frac{m}{M}$ & Energy flux & $\boldsymbol{j}_{k}^{*}=\frac{\boldsymbol{j}_{k}}{\varepsilon \sqrt{\varepsilon / M} / \sigma^{3}}$ \\
Density & $\rho^{*}=\rho \sigma^{3}$ & Thermal conductivity & $\lambda^{*}=\frac{\lambda}{k_{\mathrm{B}} \sqrt{\varepsilon / M} / \sigma^{2}}$ \\
Temperature & $T^{*}=\frac{T}{\varepsilon / k_{\mathrm{B}}}$ & Shear viscosity & $\eta^{*}=\frac{\eta}{\sqrt{\varepsilon M} / \sigma^{2}}$ \\
Pressure & $p^{*}=\frac{p}{\varepsilon / \sigma^{3}}$ & Self-diffusion coeff. & $D^{*}=\frac{D}{\sqrt{\varepsilon / M} / \sigma}$ \\
\hline
\end{tabular}

Simulation setup. The two-gradient method and the corresponding simulation setup have been discussed in detail in a recent paper of our group [10]. Therefore, only the key information is given here. A scheme of the simulation box is shown in Figure 1. It is cubic and completely filled with LJTS fluid. There are no walls, such that surface effects are avoided. Due to the periodic boundary conditions, the pressure in the box is almost constant. The mean particle density in the simulation box is prescribed. The edge length $L$ is chosen such that the box contains about 10,000 particles, leading to larger systems for low densities. To avoid finite size effects [17, 20, 21], the minimum edge length is set to $L_{\min }^{*}=24$.

The simulation box is uniformly discretized in $y$-direction. The number of bins is 48 in all simulations of the present study. The corresponding bin thickness is $\Delta y=L / 48$, resulting in the minimum thickness $\Delta y_{\min }^{*}=0.5$. The temperature gradient $\partial T / \partial y$ and the velocity gradient $\dot{\gamma}=\partial \boldsymbol{v}_{x} / \partial y$ are applied simultaneously. In the global method, the temperature gradient and the velocity gradient are set to $\partial T^{*} / \partial y^{*}=0.03$ and $\dot{\gamma}^{*}=0.03$, respectively. In the local method, the temperature gradient is increased to $\partial T^{*} / \partial y^{*}=0.1$ while the velocity gradient is not changed.

The time step is $\Delta \tau^{*}=0.002$. A simulation run consists of initially 500,000 time steps in which a steady state is established, i.e. a stable temperature and velocity gradient are built up. Subsequently, a production run of 2,000,000 time steps is conducted. Blockaveraging with a block size of 10,000 time steps is applied to determine the mean standard 
deviation for all observables that are calculated during the production run. Post-processing steps are accounted for by error propagation. The error analysis is applied for each data point. 'Mean estimated uncertainty' in the following refers to averaging of estimated errors for a given set of data points. For more details regarding the error analysis see [10].

The simulations are carried out with a substantially extended version of the molecular dynamics (MD) code ls1 mardyn [22].

\section{Overview of Literature Data on Transport Properties of LJ-Type Fluids}

There is a large number of reports on transport properties of LJ-type fluids in the literature, most of which focus on viscosity [23]. Only few studies cover a wide range of states in a systematic way [7 9]. To the best of our knowledge, there is no systematic study of transport properties of the LJTS fluid with $r_{\mathrm{c}}^{*}=2.5$.

The literature data sets of transport properties of LJ-type fluids that were considered in the present work are listed in Table 2 where they are assigned to the corresponding LJ-type. The data consist of transport properties of the LJ fluid, the truncated LJ (LJT) fluid with different cut-off radii, and the LJTS fluid with $r_{\mathrm{c}}^{*}=5.0$.

Table 2: Overview of literature data sets of transport properties of LJ-type fluids. The entire data sets consist of about 2700 data points.

\begin{tabular}{|c|c|c|c|c|c|}
\hline & LJ & $\mathrm{LJT}_{r_{\mathrm{c}}^{*}<2.5}$ & $\mathrm{LJT}_{r_{\mathrm{c}}^{*}=2.5}$ & $\mathrm{LJT}_{r_{\mathrm{c}}^{*}>2.5}$ & $\operatorname{LJTS}_{r_{\mathrm{c}}^{*}=5.0}$ \\
\hline$\lambda:$ & [7, 24-27] & 28 & [17, 2931 & 32,33 & - \\
\hline$\eta:$ & {$[7,24,25,27,34$} & - & $29,31,35,38$ & [9, 39] & [19] \\
\hline$D:$ & 17, 24, 25] & - & [40, 41] & [8, 39, 42] & - \\
\hline
\end{tabular}

A qualitative overview over the data density of all studies in the $T^{*}, \rho^{*}$-space is shown as heat maps in the Supporting Information. The distribution of the data density in the $T^{*}, \rho^{*}$-space is similar for $\lambda, \eta$, and $D$. Most data were taken in the vicinity of the vapor-liquid coexistence line, and for supercritical fluids with $T^{*} \leq 3.0$ and $\rho^{*} \geq 0.3$. In addition, for some isolated thermodynamic states in the vicinity of $\left(T^{*}=6.0, \rho^{*}=1.0\right) \mathrm{a}$ data agglomeration is found. The present study covers the whole range of $T^{*}=[0.6,10.0]$ 

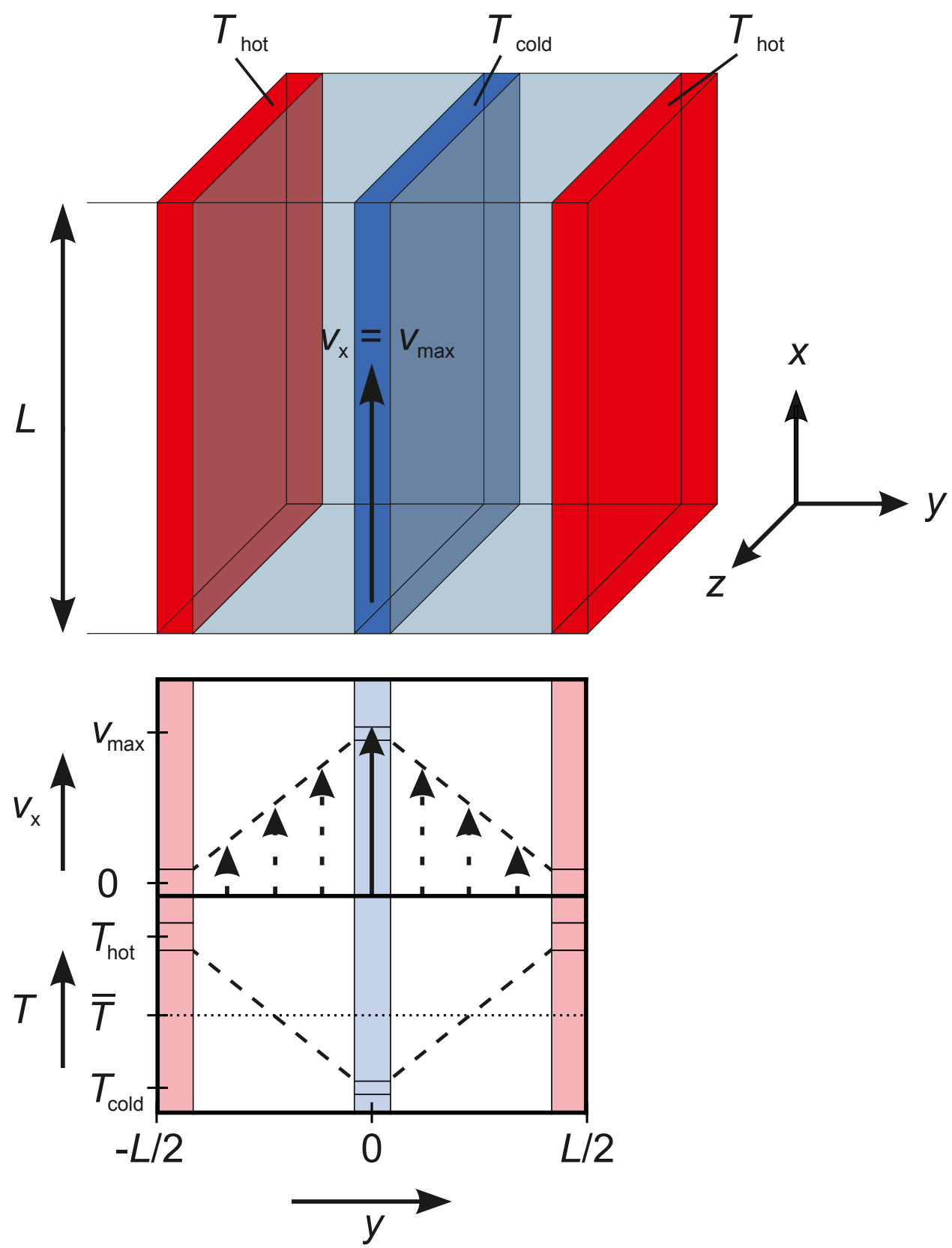

Figure 1: Scheme of the simulation setup used in the two-gradient method. The control volumes in which the temperature and the velocity are prescribed are marked in blue and red. The shear flow is in $+x$ direction. Exemplary temperature and velocity profiles are depicted at the bottom. The dashed lines and arrows indicate responses. Within the control volumes the mean temperature and the mean velocity are adjusted leading to an upper and lower bound value indicated by the solid vertical lines. 
and $\rho^{*}=[0.2,1.2]$. Hereby, our study fills the gaps in the literature data. Moreover, it provides values in regions with a low data density and extends the range where information is available.

\section{Transport Properties of the LJTS Fluid}

Data set. In the present study $\lambda, \eta$, and $D$ were determined for about 350 state points over a wide range of thermodynamic states $\left(T^{*}=[0.6,10.0]\right.$ and $\left.\rho^{*}=[0.2,1.2]\right)$. Within this range we distinguish two domains which are separated by the critical temperature of the LJTS fluid $\left(T_{\text {crit }}^{*}=1.086[43]\right)$. They are referred to as 'liquid phase' if $T^{*}<T_{\text {crit }}^{*}$ and 'supercritical region' if $T^{*} \geq T_{\text {crit }}^{*}$. With the global method 186 state points in the range of $T^{*}=[0.7,10.0]$ and $\rho^{*}=[0.2,1.2]$ were investigated. With the local method data for 159 thermodynamic states in the range of $T^{*}=[0.6,5.0]$ and $\rho^{*}=[0.2,1.0]$ are provided. The numerical results from both methods are reported in the Supporting Information. No data are reported for $\rho^{*}<0.2$ as the present simulation method is not recommended for low densities 10 .

Global method. Selected data are shown in Figure 2 together with literature values as well as correlations for the different transport properties (lines) that were obtained as described in Section 5. Three isotherms are shown in Figure 2 $T^{*}=0.8$ (blue), $T^{*}=2.0$ (green), and $T^{*}=10.0$ (red). No data are shown in the two-phase region.

In general, there is an excellent agreement between the results from the present work, the literature data, and the correlations. However, there is a systematic deviation between the correlation and the simulation data (both from the present work and from the literature) for $\eta$ at $\rho^{*} \lesssim 0.4$.

The mean estimated uncertainty of the full set of simulation data determined with the global method in the present work for $(\lambda, \eta$, and $D)$ is $(3.5 \%, 2.1 \%, 0.7 \%)$ for the liquid phase, and $(2.6 \%, 3.4 \%, 0.7 \%)$ for the supercritical region. Thus, the accuracy of the global method is similar to that of established methods, cf. [8, 9, 17, 32].

Local method. In Figure 3 selected results from the local method are compared to results from the global method, as well as to the correlations. The plotted values are in a small band of pressures around specific isobars: $p^{*}=1.5 \pm 10 \%$ (blue), $p^{*}=4.0 \pm 10 \%$ (green), and $p^{*}=8.0 \pm 10 \%$ (red). 

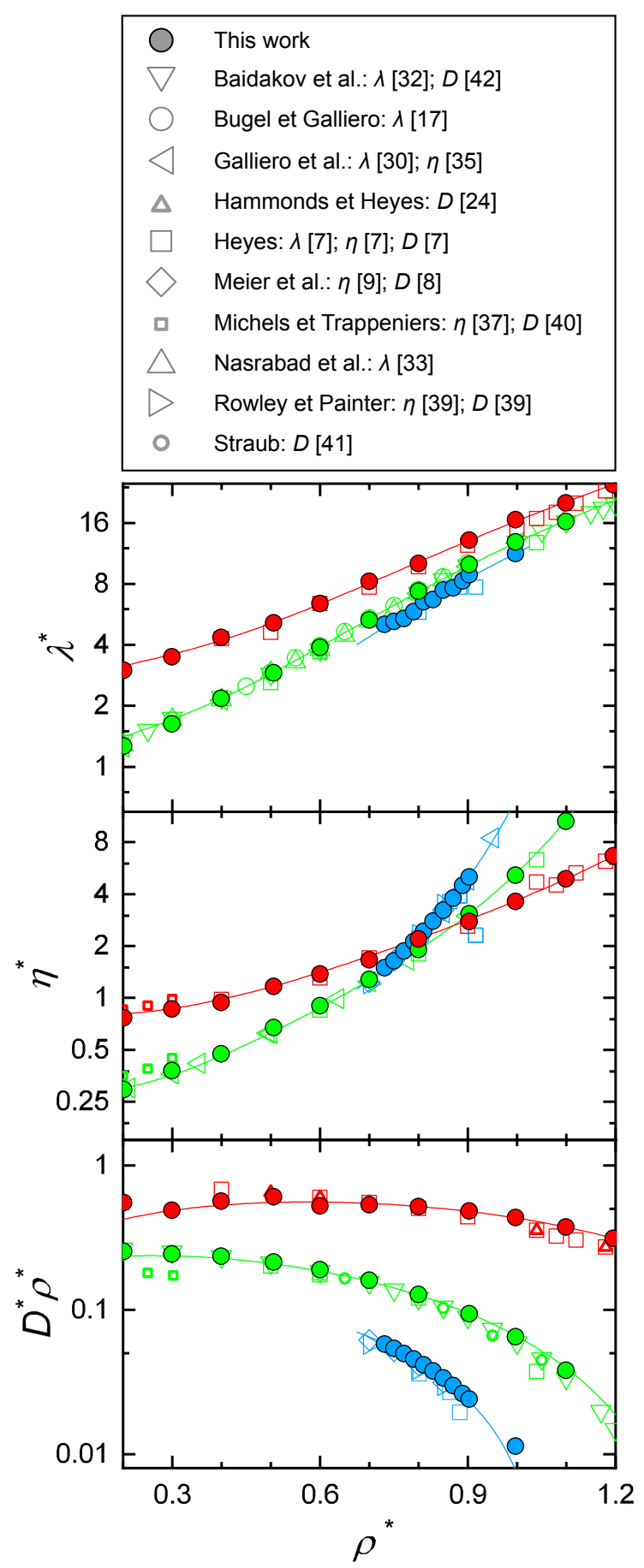

Figure 2: Transport properties of LJ-type fluids. Comparison of data from the present work determined with the global method, literature data, and correlations from the present work (lines) for three isotherms: $T^{*}=0.8$ (blue), $T^{*}=2.0$ (green), and $T^{*}=10.0$ (red). The estimated uncertainties of the results from the present work are within the symbol size. 


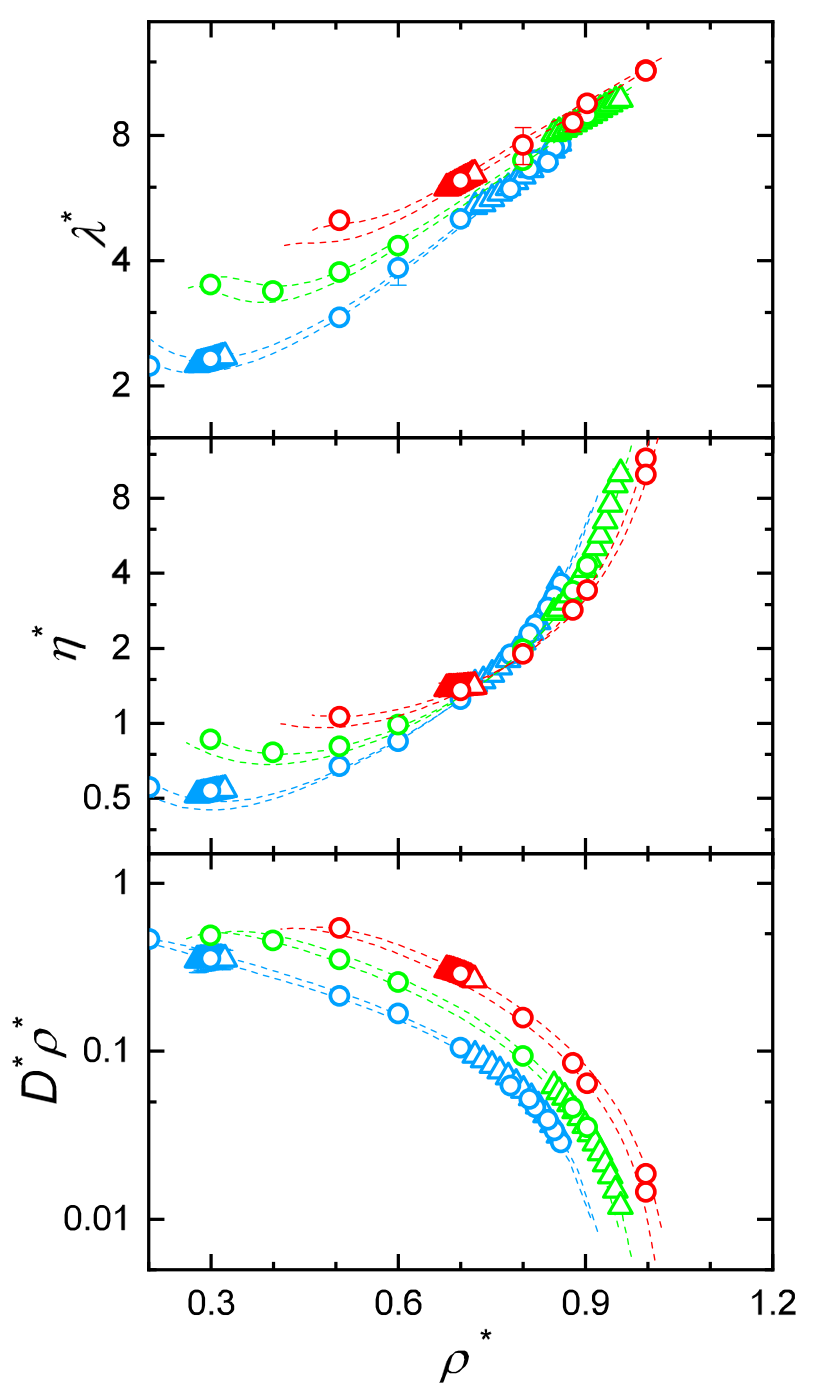

Figure 3: Transport properties of LJ-type fluids. Comparison of data from the present work determined with different methods: global method (circles), and local method (triangles). Data in the vicinity of three isobars is shown: $p^{*}=1.5$ (blue), $p^{*}=4.0$ (green), and $p^{*}=8.0$ (red). The upper bound $\left(p^{*}+10 \%\right)$ and the lower bound $\left(p^{*}-10 \%\right)$ of the three isobars are calculated using the correlations from the present work. The estimated uncertainties of the data points are depicted by the error bars which are mostly within the symbol size. 
The results from the local method, the global method, and the correlations agree well.

The mean estimated uncertainty of the full set of simulation data determined with the local method in the present work for $(\lambda, \eta$, and $D)$ is $(1.0 \%, 1.4 \%, 1.5 \%)$ for the liquid phase, and $(0.8 \%, 2.9 \%, 5.7 \%)$ for the supercritical region. Compared to the global method the mean estimated uncertainties of the local method are as follows: 1) For $\lambda$ the uncertainty is lower. This is due to the large heat flux coming from the large temperature gradient that is applied inherently. 2) For $\eta$ the uncertainty is similar to that of the global method as the velocity gradient is similar to that of the global method. 3) For $D$ the uncertainty is much higher. This is due to poorer statistics resulting from the lower number of fluid particles that is considered for averaging $D$, cf. [10]. However, a direct comparison of the mean estimated uncertainties is slightly biased by the different thermodynamic states that were studied with each of the two methods. The global method was applied for studying the entire range of states whereas the local method was mainly applied in regions that are particularly easy to study. More details are given in the Supporting Information.

The local method is considerably more efficient than the global method and probably also more efficient than any other method for determining transport properties from molecular simulations: data for $\lambda, \eta$, and $D$ for about 15 different thermodynamic states are obtained in a single simulation run.

\section{Correlations of the Transport Properties}

Based on the data set determined using the global method $(N=186)$ simple empirical correlations describing the three transport properties were established. They are presented in Eqs. (3), (4) \& (5).

$$
\begin{gathered}
\lambda^{*}\left(T^{*}, \rho^{*}\right)=\sum_{i} \sum_{j} a_{i j} T^{* i} \rho^{* j} \\
\eta^{*}\left(T^{*}, \rho^{*}\right)=\left(b_{0} \rho^{*}\right) \exp \left[\left(c_{0} \rho^{*}+\left(d_{0} \rho^{* 4}-\rho^{*}\right) T^{*(-1)}\right)\right]+\sum_{i=1}^{5} e_{i} T^{* u_{i}} \rho^{* v_{i}} \\
D^{*} \rho^{*}\left(T^{*}, \rho^{*}\right)=\sum_{i} \sum_{j} f_{i j} T^{* i} \rho^{* j}
\end{gathered}
$$


The parameters of the correlations were determined by least squares regression from the simulation data and are valid for $T^{*}=[0.7,10.0]$ and $\rho^{*}=[0.2,1.2]$. The parameters of the correlations shown in Eqs. (3), (4) \& (5) are summarized in Table 3.

Table 3: Regression coefficients used for the correlations of the different transport properties, cf. Eqs. (3), (4) \& (5).

\begin{tabular}{|c|c|c|c|c|c|c|c|c|}
\hline & $i$ & $j$ & $a_{i j}$ & & & $i$ & $j$ & $f_{i j}$ \\
\hline \multirow[t]{16}{*}{$\lambda^{*}$} & 0 & 0 & -0.08633 & & $D^{*} \rho^{*}$ & 0 & 0 & -0.002737 \\
\hline & 0 & 1 & 4.377 & & & 0 & 1 & 0.3043 \\
\hline & 0 & 2 & -14.25 & & & 0 & 2 & -0.6629 \\
\hline & 0 & 3 & 25.49 & & & 0 & 3 & 0.3294 \\
\hline & 0 & 4 & -5.527 & & & 1 & 0 & 0.1173 \\
\hline & 1 & 0 & 0.5723 & & & 1 & 1 & 0.006592 \\
\hline & 1 & 1 & 0.6753 & & & 1 & 2 & -0.07464 \\
\hline & 1 & 2 & 0.7847 & & & 2 & 0 & -0.009532 \\
\hline & 1 & 3 & -0.03917 & & & 2 & 1 & 0.009238 \\
\hline & 2 & 0 & -0.127 & & & & & \\
\hline & 2 & 1 & -0.1697 & & & & & \\
\hline & 2 & 2 & -0.04694 & & & & & \\
\hline & 3 & 0 & 0.02022 & & & & & \\
\hline & 3 & 1 & 0.01147 & & & & & \\
\hline & 4 & 0 & -0.001088 & & & & & \\
\hline & $i$ & $b_{i}$ & $c_{i}$ & $d_{i}$ & $e_{i}$ & $u_{i}$ & $v_{i}$ & \\
\hline \multirow[t]{6}{*}{$\eta^{*}$} & 0 & 0.5736 & 1.38 & 2.438 & - & - & - & \\
\hline & 1 & - & - & - & 0.1786 & 0 & 0 & \\
\hline & 2 & - & - & - & 0.06529 & 1 & 0 & \\
\hline & 3 & - & - & - & -1.158 & 0 & 1 & \\
\hline & 4 & - & - & - & 1.347 & 0 & 2 & \\
\hline & 5 & - & - & - & 0.3194 & -1 & 1 & \\
\hline
\end{tabular}

The simulation results and the correlations for the three studied transport properties 
are compared in deviation plots in Figure 4. Almost all simulation data are represented by the correlations with deviations below $\pm 10 \%$. The largest deviations for $\lambda$ and $\eta$ are found close to the critical point of the LJTS fluid $\left(T_{\text {crit }}^{*}=1.086\right.$ and $\rho_{\text {crit }}^{*}=0.319$ [43]). The largest deviations for $D \rho$ are found for states with a low density and the highest temperature $T^{*}=10.0$, and are due to numerical issues.

Deviations of the correlation and the simulation data are quantified here using the average absolute deviation (AAD)

$$
\mathrm{AAD}=\frac{1}{N} \sum_{i=1}^{N}\left|1-\frac{x_{i, \text { global }}}{x_{i, \text { corr }}}\right|,
$$

and the bias

$$
\text { bias }=\frac{1}{N} \sum_{i=1}^{N}\left(1-\frac{x_{i, \text { global }}}{x_{i, \text { corr }}}\right) .
$$

Here, $x_{i \text {,global }}$ stands for the transport property $\lambda$, $\eta$, or $D \rho$ at a state $i$ determined using the global method. $x_{i \text {,corr }}$ is the corresponding value determined using the correlation. The numbers for AAD and bias for $\lambda, \eta$, and $D \rho$ are reported in Table 4 for two data sets obtained with the global method: the full data set and data with $\rho^{*} \geq \rho_{\text {crit }}^{*}$, i.e. excluding states with low densities.

Table 4: Statistical evaluation of the correlations for $\lambda, \eta$ and $D \rho$ (cf. Eqs. (3), 44 \& (5)) by comparison to simulation results determined in the present work using the global method. Two sets of simulation results are shown that differ regarding the minimal density $\rho_{\min }^{*}$ which is considered for the comparison. As reference values also information on the mean estimated uncertainty (MEU) of the simulation data is given.

\begin{tabular}{cccccc}
\hline \hline & $\rho_{\text {min }}^{*}$ & $N$ & $\operatorname{AAD}(\%)$ & $\operatorname{bias}(\%)$ & $\operatorname{MEU}(\%)$ \\
\hline$\lambda^{*}$ & 0.2 & 186 & 2.37 & -0.002 & $2.6-3.5$ \\
& $\rho_{\text {crit }}^{*}$ & 169 & 1.98 & -0.271 & $2.6-3.5$ \\
\hline$\eta^{*}$ & 0.2 & 186 & 1.93 & 0.226 & $2.1-3.4$ \\
& $\rho_{\text {crit }}^{*}$ & 169 & 1.19 & -0.074 & $2.1-3.4$ \\
\hline$D^{*} \rho^{*}$ & 0.2 & 186 & 2.02 & -0.693 & 0.7 \\
& $\rho_{\text {crit }}^{*}$ & 169 & 1.49 & -0.416 & 0.7 \\
\hline \hline
\end{tabular}



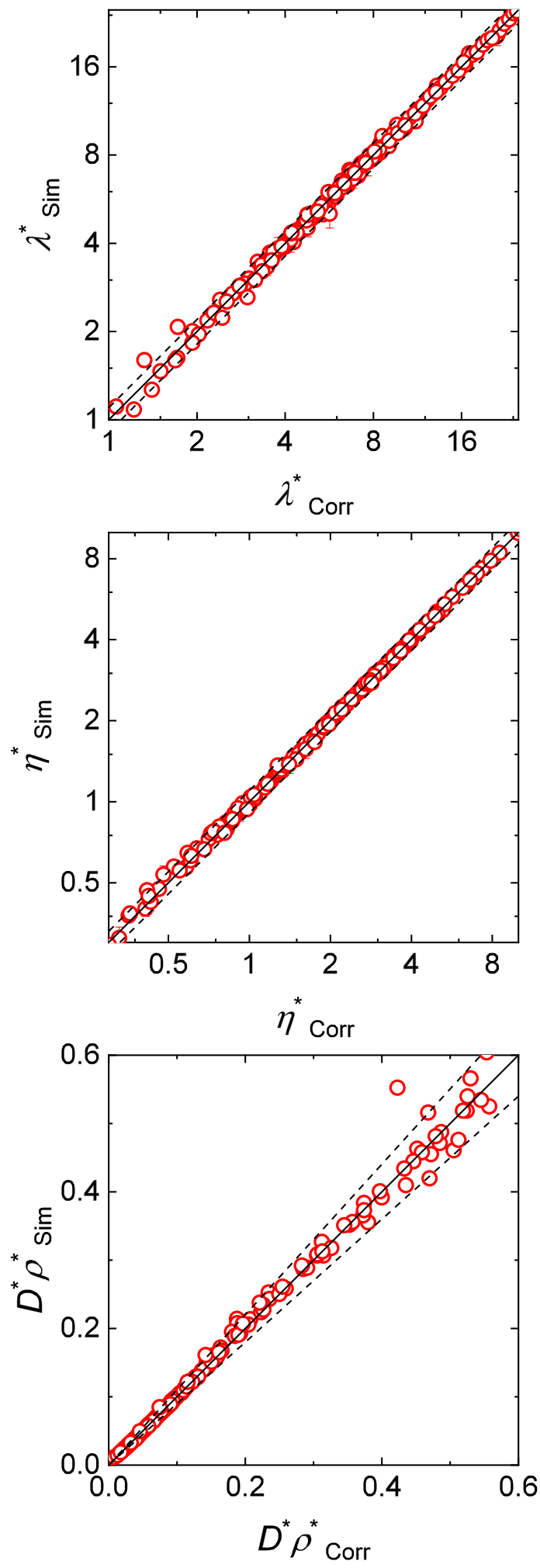

Figure 4: Deviation plots comparing the transport properties calculated using the global method with the correlations of the different transport properties. The whole data set with $N=186$ thermodynamic states is considered and depicted with the red circles. The dashed lines indicate a band of $\pm 10 \%$ around the solid line. The estimated uncertainties of the results from the present work are depicted by the error bars which are mostly within the symbol size. 
The results presented in Table 4 show that the simulation data of $\lambda$ and $\eta$ are described with almost no bias generally within their uncertainty, while for $D \rho$ the AAD is slightly larger than the uncertainty of the simulation data. However, for liquid-like densities $\left(\rho^{*} \geq \rho_{\text {crit }}^{*}\right)$ the AAD is of the order of the mean estimated uncertainty of the simulation data set.

The present correlations are established mainly for facilitating comparisons within the present study. There are other correlations for different transport properties of LJ-type fluids in the literature: $\lambda$ [17, 30, $\eta$ [35, 39], and $D$ [39]. Most of them were established on narrower data bases, and they are generally more complicated, e.g. they require solving collision integrals [44]. A comparison of the present correlation and the correlations from the literature is given in the Supporting Information. It shows that the simple correlations presented here have a generally similar accuracy as those reported in the literature.

\section{Comparison of the Correlations with Results from the Local Method and} Literature Data

Local method. In Figure 5 the results for $\lambda, \eta$, and $D$ from the local method $(N=159)$ are compared to the correlations of the different transport properties that were established based on the data from the global method. The colors correspond to the mean temperature of the respective data series. Summary information of the comparison between the simulation data from the local method and the correlations is given in Table 5.

Figure 5 shows that the results from the local method agree well with the results from the correlations, and hence also with those from the global method. However, slight systematic deviations for each of the three transport properties are found for low densities $\left(\rho^{*} \approx 0.2\right)$, cf. the single yellow data series that deviates most. The generally good accordance is underpinned by the results from Table 5. Even though the correlations were not adjusted to the data from the local method, the average deviations for $\eta$ and $D \rho$ are only slightly higher than those given in Table 4. For $\lambda$ the AAD for the results from the local method is even smaller than that obtained for the results from the global method. This is due to the higher accuracy of the local method for that property, but it also proves the high quality of the correlation of $\lambda$. These are very favorable findings, especially when considering that with the local method data for $\lambda, \eta$, and $D$ at about 15 state points can 

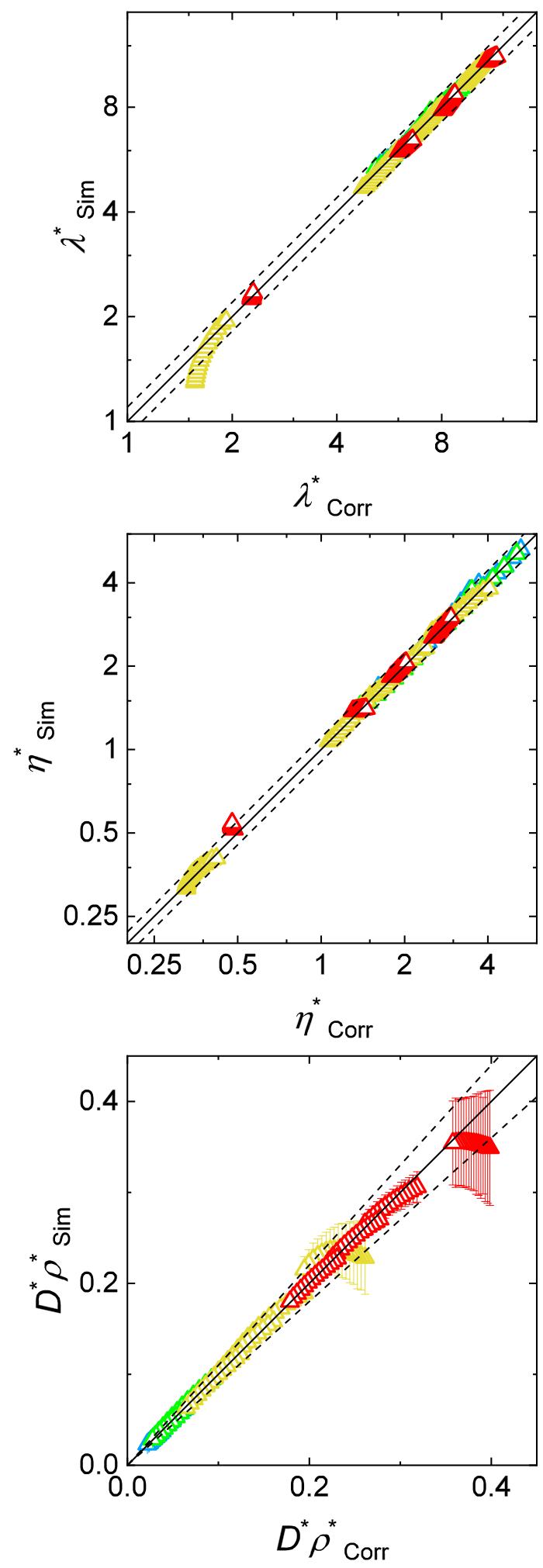

Figure 5: Deviation plots comparing the transport properties calculated using the local method with the correlations of the different transport properties obtained from the global method. The data points are colored according to the mean temperature of the respective data series. For $\bar{T}^{*}=\{0.8 ; 1.0 ; 2.0 ; 4.0\}$ they are depicted in \{blue; green; yellow; red\}. The dashed lines indicate a band of $\pm 10 \%$ around the solid line. The estimated uncertainties of the results from the present work are depicted by the error bars, where they exceed the symbol size. 
Table 5: Statistical evaluation of the correlations for $\lambda, \eta$ and $D \rho$ (cf. Eqs. (3), (4) \& (5)) by comparison to simulation results determined in the present work using the local method. Two sets of simulation results are shown that differ regarding the minimal density $\rho_{\min }^{*}$ which is considered for the comparison. As reference values also information on the mean estimated uncertainty (MEU) of the simulation data is given.

\begin{tabular}{cccccc}
\hline \hline & $\rho_{\text {min }}^{*}$ & $N$ & $\operatorname{AAD}(\%)$ & $\operatorname{bias}(\%)$ & $\operatorname{MEU}(\%)$ \\
\hline$\lambda^{*}$ & 0.2 & 159 & 1.72 & 0.528 & $0.8-1.0$ \\
& $\rho_{\text {crit }}^{*}$ & 137 & 1.19 & -0.122 & $0.8-1.0$ \\
\hline$\eta^{*}$ & 0.2 & 159 & 2.77 & -2.192 & $1.4-2.9$ \\
& $\rho_{\text {crit }}^{*}$ & 137 & 2.22 & -1.656 & $1.4-2.9$ \\
\hline$D^{*} \rho^{*}$ & 0.2 & 159 & 3.10 & -0.676 & $1.5-5.7$ \\
& $\rho_{\text {crit }}^{*}$ & 137 & 2.47 & -1.743 & $1.5-5.7$ \\
\hline \hline
\end{tabular}

be obtained in a single simulation run, that is not distinctly more costly than runs with other well-established methods.

Literature data. As mentioned before, to the best of our knowledge no comprehensive data set for the LJTS fluid from the literature is available for a comparison. However, it has been stated that the truncation has little influence on the transport properties as long as $r_{\mathrm{c}}^{*} \geq 2.5[6,8$, , 9, 17- 19]. Thus, in the following, the correlations of the different transport properties that were established in the present work for the LJTS fluid are compared to literature data for different LJ-type fluids, cf. Table 2 .

For the evaluation of the influence of the LJ-type on the transport properties representative state points in the liquid and the supercritical regime were chosen, for which data of at least two different LJ-type fluids were available. Details on the selection are given in the Supporting Information. The selected data are called 'reduced literature data set' in the following. A detailed list of it is given in the Supporting Information.

Figure 6 shows the deviations between values the from reduced literature data set for different LJ-type fluids and the correlations of the different transport properties. In general, the reduced literature data set is in good accordance with the correlations. Regarding $\lambda$ and $\eta$, no influence of the truncation and shifting of the LJ potential is observed. The 
data scatters mainly within a band of $\pm 10 \%$. The only clear trend that is observed is for the rare data on $D \rho$, where the numbers for the LJ fluid are consistently slightly lower than the prediction from the correlation and the simulation data for LJT fluids. The aforementioned findings are underpinned by Table 6 . The bias is generally well below the AAD which confirms the absence of systematic deviations. The only exception are the results for $D \rho$ for the LJ fluid for which the bias and the AAD are similar. This confirms the findings from Figure 6 but might be influenced by the low number of data points of the LJ fluid that were included in the reduced literature data set.

Table 6: Statistical evaluation of the influence of truncation and shifting of the LJ potential on the value of $\lambda, \eta$, and $D$. Values from the reduced literature data set of different LJ-type fluids are compared with the correlations in the liquid phase and the supercritical regime $\left(T^{*}=[1.0,6.0], \rho^{*}=[0.2,1.2]\right) . \rho_{\min }^{*}=0.2$ in all cases.

\begin{tabular}{ccccc}
\hline \hline & LJ-type & $N$ & AAD $(\%)$ & bias (\%) \\
\hline$\lambda^{*}$ & LJ & 15 & 5.04 & 3.489 \\
& LJT $\left(r_{\mathrm{c}}^{*}=2.5\right)$ & 20 & 1.80 & -0.467 \\
& $\operatorname{LJT}\left(r_{\mathrm{c}}^{*}>2.5\right)$ & 16 & 1.83 & 1.035 \\
\hline$\eta^{*}$ & $\mathrm{LJ}$ & 15 & 3.15 & -0.807 \\
& $\operatorname{LJT}\left(r_{\mathrm{c}}^{*}=2.5\right)$ & 13 & 5.97 & -2.479 \\
& $\operatorname{LJT}\left(r_{\mathrm{c}}^{*}>2.5\right)$ & 40 & 3.92 & -0.377 \\
& $\operatorname{LJTS}\left(r_{\mathrm{c}}^{*}=5.0\right)$ & 19 & 3.89 & -2.833 \\
\hline$D^{*} \rho^{*}$ & $\mathrm{LJ}$ & 12 & 5.87 & 5.866 \\
& $\operatorname{LJT}\left(r_{\mathrm{c}}^{*}>2.5\right)$ & 14 & 3.66 & 1.602 \\
\hline \hline
\end{tabular}

In Figure 6 and Table 6 the correlations were compared only to values from the reduced literature data set, i.e. at representative state points. In the following, a similar comparison is carried out for the entire set of available data, see Table 2. Some obvious outliers were removed and the comparison is restricted to the range of the validity of the present correlation, i.e. to $T^{*} \geq 0.7$ and $\rho^{*} \geq 0.2$. The final data set that was used contains 630 values for $\lambda, 755$ values for $\eta$, and 648 values for $D$. To the best of our understanding this is the most comprehensive comparison ever done for transport properties of LJ-type fluids. 

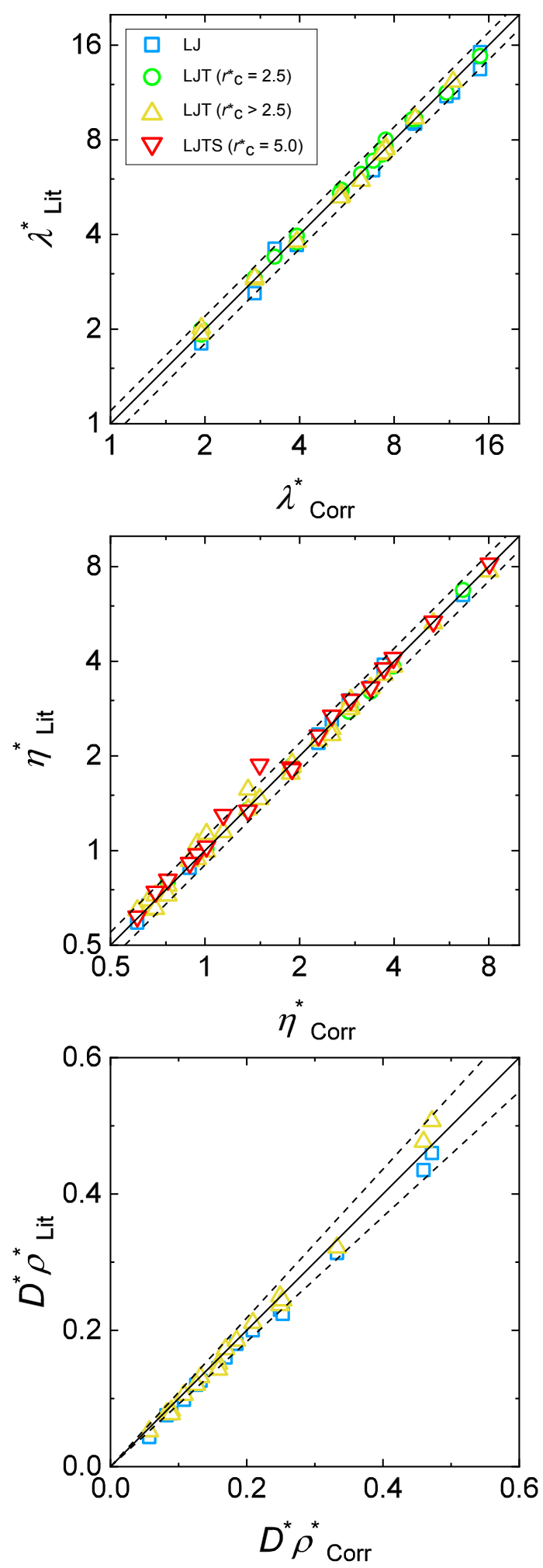

Figure 6: Deviation plots comparing a set of selected literature data (see text) with the correlations developed in the present work. The investigated state points are in the liquid and the supercritical regime $\left(T^{*}=[1.0,6.0], \rho^{*}=[0.2,1.2]\right)$. The data points are colored according to the LJ-type fluid used for their determination, cf. legend. The dashed lines indicate a band of $\pm 10 \%$ around the solid line. 
The results are shown in Figure 7. Thereby, the aforementioned findings are confirmed. The literature data for $\lambda$ and $\eta$ agree very well with the correlation over the whole range of thermodynamic states that is considered here. No significant influence of the LJ-type is observed. Interestingly, this is different for $D \rho$, where the literature values of the LJ fluid are mostly smaller than the results from the correlation, while the data of the LJT fluid with $r_{\mathrm{c}}^{*} \geq 2.5$ is represented well by the correlation. It scatters mainly within a band of $\pm 10 \%$.

The literature data depicted in Figure 7 is quantitatively analyzed by means of the AAD and the bias as reported in Table 7 . Again, for $\lambda$ and $\eta$, the bias is significantly lower than the AAD. This does not hold for $D \rho$ and supports the statement that a truncation of the LJ potential increases $D$. All in all, the present comparison confirms that the transport properties $\lambda$ and $\eta$ of the LJ-type fluids are not significantly influenced by either the truncation or the shifting as long as the cut-off radius $r_{\mathrm{c}}^{*}$ is above about 2.5. However, we find that a truncation of the LJ potential increases $D$, i.e the particle mobility.

Table 7: Results from the comparison of the complete literature data set on transport properties of LJtype fluids with the correlations for the LJTS fluid from the present work. The last row of each sub-block in the table represents a comparison of the correlations with single references that are discussed in more detail in the Supporting Information.

\begin{tabular}{ccccc}
\hline \hline & $\rho_{\text {min }}^{*}$ & $N$ & AAD (\%) & bias $(\%)$ \\
\hline$\lambda^{*}$ & 0.2 & 630 & 3.74 & 0.822 \\
& $\rho_{\text {crit }}^{*}$ & 588 & 3.43 & 0.996 \\
{$[17,[32]$} & $\rho_{\text {crit }}^{*}$ & 220 & 2.22 & -0.301 \\
\hline$\eta^{*}$ & 0.2 & 755 & 4.70 & 0.402 \\
& $\rho_{\text {crit }}^{*}$ & 680 & 4.29 & 0.905 \\
{$[9]$} & $\rho_{\text {crit }}^{*}$ & 187 & 2.76 & 1.609 \\
\hline$D^{*} \rho^{*}$ & 0.2 & 648 & 8.31 & 6.097 \\
& $\rho_{\text {crit }}^{*}$ & 566 & 7.84 & 6.994 \\
\hline 8 & $\rho_{\text {crit }}^{*}$ & 183 & 3.84 & 2.743 \\
\hline \hline
\end{tabular}



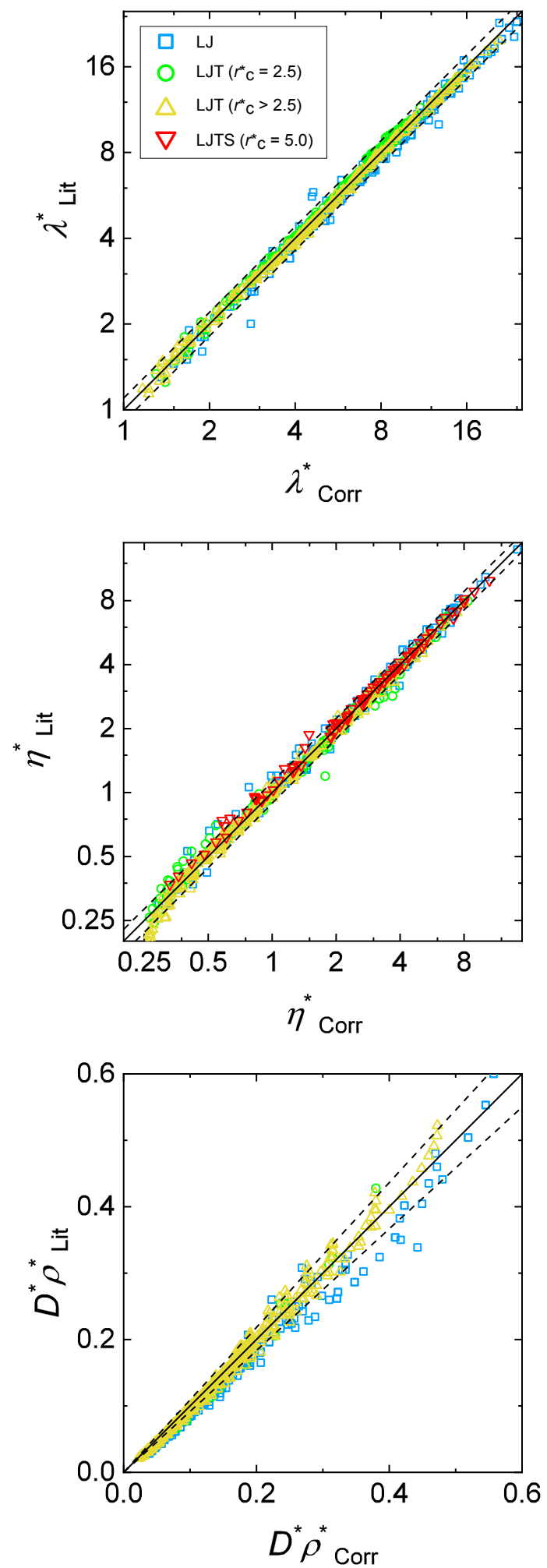

Figure 7: Deviation plots comparing the complete literature data set (cf. Table 2) with the correlations developed here. The investigated state points are in the liquid and the supercritical regime $\left(T^{*}=[0.6,10.0], \rho^{*}=[0.2,1.2]\right)$. The data points are colored according to the LJ-type fluid used for their determination, cf. legend. The dashed lines indicate a band of $\pm 10 \%$ around the solid line. 


\section{Conclusion}

In the present study, the transport properties thermal conductivity $\lambda$, shear viscosity $\eta$, and self-diffusion coefficient $D$ of the LJTS fluid with $r_{\mathrm{c}}^{*}=2.5$ are investigated in a wide range of thermodynamic states $\left(T^{*}=[0.7,10.0], \rho^{*}=[0.2,1.2]\right)$. The two-gradient method is applied for the simulations. In that method, different transport properties are obtained from a single simulation run. There are two variants, in which the twogradient method can be applied. In the global method one state point is studied in one simulation run. In the local method data for several temperatures are collected in one single simulation run. 186 state points were studied here with the global method and 159 with the local method. It is shown that the results from the global and the local method agree well. This confirms the applicability of the computationally highly efficient local method. Altogether, the only comprehensive data base for the LJTS fluid with $r_{\mathrm{c}}=2.5 \sigma$ is provided. More than 1000 new data points are provided for transport properties of the LJTS fluid, for which to the best of our knowledge previously no data set was available. In addition, the number of data points for the whole class of LJ-type fluids is significantly increased. The collection of such a large amount of data was only possible due to the high efficiency of the two-gradient method, especially in its variant 'local method'.

Based on the data determined with the global method simple empirical correlations for $\lambda, \eta$, and $D \rho$ were developed. The results describe the data provided in the present work very well, however with some restrictions at low densities. The results from the correlations of the different transport properties are also compared to literature data of LJ-type fluids. The comparison confirms that the truncation of the LJ potential has no significant influence on the transport properties $\lambda$ and $\eta$ for $r_{\mathrm{c}} \geq 2.5 \sigma$. We could show that this is not valid for $D$, where a truncation of the LJ potential leads to larger values of $D$. It would be interesting to use the comprehensive new data set provided in the present work for studying physically motivated descriptions of transport properties, especially the entropy scaling [45,47]. 


\section{Acknowledgement}

The authors gratefully acknowledge financial support by the DFG within IRTG 2057 "Physical Modeling for Virtual Manufacturing Systems and Processes". The simulations were carried out on the HazelHen at High Performance Computing Center Stuttgart (HLRS) under the grant MMHBF2, on elwe at Regional University Computing Center Kaiserslautern (RHRK) under the grant TUKL-TLMV as well as on the SuperMuc at Leibniz Supercomputing Centre (LRZ) Garching under the grant SPARLAMPE (pr48te). The present research was conducted under the auspices of the Boltzmann-Zuse Society for Computational Molecular Engineering (BZS). 


\section{References}

[1] M. P. Allen, D. J. Tildesley, Computer simulation of liquids, Oxford Science Publications, New York, 1989.

[2] G. Karniadakis, A. Beskok, N. Aluru, Microflows and Nanoflows, Vol. Second Edition, Springer-Verlag, New York, 2005.

[3] K. M. Dyer, B. M. Pettitt, G. Stell, Systematic investigation of theories of transport in the Lennard-Jones fluid, The Journal of Chemical Physics 126 (3) (2007) 034502. doi:10.1063/1.2424714.

URL http://aip.scitation.org/doi/10.1063/1.2424714

[4] G. Rutkai, M. Thol, R. Span, J. Vrabec, How well does the Lennard-Jones potential represent the thermodynamic properties of noble gases?, Molecular Physics 115 (9-12) (2017) 1104-1121. doi:10.1080/00268976.2016.1246760.

URL https://www.tandfonline.com/doi/full/10.1080/00268976.2016. 1246760

[5] J. P. Ewen, D. M. Heyes, D. Dini, Advances in nonequilibrium molecular dynamics simulations of lubricants and additives, Friction (Md) (2018) 10.1007/s40544-0180207-9. doi:10.1007/s40544-018-0207-9.

URL http://link.springer .com/10.1007/s40544-018-0207-9

[6] C. Hoheisel, Bulk viscosity of model fluids. A comparison of equilibrium and nonequilibrium molecular dynamics results, The Journal of Chemical Physics 86 (4) (1987) 2328-2333. doi:10.1063/1.452131.

URL http://link.aip.org/link/JCPSA6/v86/i4/p2328/s1\{\&\}Agg=doihttp: //aip.scitation.org/doi/10.1063/1.452131

[7] D. M. Heyes, Transport coefficients of Lennard-Jones fluids: A molecular-dynamics and effective-hard-sphere treatment, Physical Review B 37 (10) (1988) 5677-5696. doi:10.1103/PhysRevB.37.5677.

URL https://link.aps.org/doi/10.1103/PhysRevB.37.5677 
[8] K. Meier, A. Laesecke, S. Kabelac, Transport coefficients of the Lennard-Jones model fluid. II Self-diffusion, The Journal of Chemical Physics 121 (19) (2004) 9526-9535. doi:10.1063/1.1786579.

URL http://aip.scitation.org/doi/10.1063/1.1786579

[9] K. Meier, A. Laesecke, S. Kabelac, Transport coefficients of the Lennard-Jones model fluid. I. Viscosity, The Journal of Chemical Physics 121 (8) (2004) 3671-3687. doi: $10.1063 / 1.1770695$.

URL http://aip.scitation.org/doi/10.1063/1.1770695

[10] M. P. Lautenschlaeger, M. Horsch, H. Hasse, Simultaneous Determination of Thermal Conductivity and Shear Viscosity Using Two-Gradient Non-Equilibrium Molecular Dynamics Simulations, Molecular Physicsdoi:10.1080/00268976.2018.1504134. URL https://doi .org/10.1080/00268976.2018.1504134

[11] A. Tenenbaum, G. Ciccotti, R. Gallico, Stationary nonequilibrium states by molecular dynamics. Fourier's law, Physical Review A 25 (5) (1982) 2778-2787. doi:10.1103/ PhysRevA.25.2778.

[12] A. Tenenbaum, Local equilibrium in stationary states by molecular dynamics, Physical Review A 28 (5) (1983) 3132-3133.

[13] S. de Groot, P. Mazur, Non-Equilibrium Thermodynamics, Dover Publication, New York, 1984.

[14] F. Bresme, J. Armstrong, Note: Local thermal conductivities from boundary driven non-equilibrium molecular dynamics simulations, The Journal of Chemical Physics 140 (1) (2014) 016102. doi:10.1063/1.4858434.

URL http://www.ncbi.nlm.nih.gov/pubmed/24410242http://aip.scitation. org/doi/10.1063/1.4858434

[15] F. Bresme, Non-equilibrium simulations of fluids under thermal gradients: local properties and coupling effects, in: Joint European Thermodynamics Conference, Vol. 1, 2017, pp. 25-28. 
[16] H. Matsubara, G. Kikugawa, T. Bessho, S. Yamashita, T. Ohara, Non-equilibrium molecular dynamics simulation as a method of calculating thermodynamic coefficients, Fluid Phase Equilibria 421 (2016) 1-8. doi:10.1016/j.fluid.2016.03.019. URL http://dx.doi.org/10.1016/j.fluid.2016.03.019

[17] M. Bugel, G. Galliéro, Thermal conductivity of the Lennard-Jones fluid: An empirical correlation, Chemical Physics 352 (1-3) (2008) 249-257. doi:10.1016/j.chemphys. 2008.06 .013 .

URL http://linkinghub.elsevier.com/retrieve/pii/S0301010408003753

[18] C. Hoheisel, R. Vogelsang, M. Schoen, Bulk viscosity of the Lennard-Jones fluid for a wide range of states computed by equilibrium molecular dynamics, The Journal of Chemical Physics 87 (12) (1987) 7195-7198. doi:10.1063/1.453362.

URL http://scitation.aip.org/content/aip/journal/jcp/87/12/10.1063/1. 453362http://aip.scitation.org/doi/10.1063/1.453362

[19] V. R. Vasquez, E. A. Macedo, M. S. Zabaloy, Lennard-Jones Viscosities in Wide Ranges of Temperature and Density: Fast Calculations Using a SteadyâĂŞState Periodic Perturbation Method, International Journal of Thermophysics 25 (6) (2004) 1799-1818. doi:10.1007/s10765-004-7736-3.

URL http://link. springer .com/10.1007/s10765-004-7736-3

[20] K. Binder, J. Horbach, W. Kob, W. Paul, F. Varnik, Molecular dynamics simulations, Journal of Physics: Condensed Matter 16 (5) (2004) S429-S453. arXiv:arXiv:1409.6842v1, doi:10.1088/0953-8984/16/5/006

URL http://stacks .iop.org/0953-8984/16/i=5/a=006?key=crossref. 05e092a2ecfbed5e925b2c5e582bd78c

[21] J. Nichele, I. Borges, A. B. Oliveira, L. S. Alves, Molecular dynamics simulations of momentum and thermal diffusion properties of near-critical argon along isobars, The Journal of Supercritical Fluids 114 (2016) 46-54. doi:10.1016/j.supflu.2016.04.004.

URL http://dx.doi.org/10.1016/j.supflu.2016.04.004http://linkinghub. elsevier.com/retrieve/pii/S089684461630078X 
[22] C. Niethammer, S. Becker, M. Bernreuther, M. Buchholz, W. Eckhardt, A. Heinecke, S. Werth, H.-J. Bungartz, C. W. Glass, H. Hasse, J. Vrabec, M. Horsch, ls1 mardyn : The Massively Parallel Molecular Dynamics Code for Large Systems, Journal of Chemical Theory and Computation 10 (10) (2014) 4455-4464. doi:10.1021/ct500169q.

URL http://pubs .acs .org/doi/abs/10.1021/ct500169q

[23] B. D. Todd, P. J. Daivis, Homogeneous non-equilibrium molecular dynamics simulations of viscous flow: techniques and applications, Molecular Simulation 33 (3) (2007) 189-229. doi:10.1080/08927020601026629.

URL http://www . tandfonline.com/doi/abs/10.1080/08927020601026629

[24] K. D. Hammonds, D. M. Heyes, Transport coefficients of model simple liquids. A molecular-dynamics study and effective hard-sphere analysis, Journal of the Chemical Society, Faraday Transactions 284 (6) (1988) 705. doi:10.1039/f29888400705.

URL http://xlink.rsc . org/?DOI=f29888400705

[25] D. Heyes, J. Powles, Information theory applied to the transport coefficients of Lennard-Jones fluids, Molecular Physics 71 (4) (1990) 781-800. doi:10.1080/ 00268979000102111 .

URL http://www . tandfonline.com/doi/abs/10.1080/00268979000102111

[26] D. Levesque, L. Verlet, Molecular dynamics calculations of transport coefficients, Molecular Physics 61 (1) (1987) 143-159. arXiv:https://doi.org/10.1080/ 00268978700101041 , doi:10.1080/00268978700101041.

URL https://doi .org/10.1080/00268978700101041

[27] R. D. Mountain, System size and control parameter effects in reverse perturbation nonequilibrium molecular dynamics, The Journal of Chemical Physics 124 (10) (2006) 104109. doi:10.1063/1.2178340.

URL http://aip.scitation.org/doi/10.1063/1.2178340

[28] R. Vogelsang, C. Hoheisel, G. Ciccotti, Thermal conductivity of the lennardâĂŘjones liquid by molecular dynamics calculations, The Journal of Chemical Physics 86 (11) 
(1987) 6371-6375. arXiv:https://doi.org/10.1063/1.452424, doi:10.1063/1. 452424

URL https://doi.org/10.1063/1.452424

[29] P. Borgelt, C. Hoheisel, G. Stell, Exact molecular dynamics and kinetic theory results for thermal transport coefficients of the Lennard-Jones argon fluid in a wide region of states, Physical Review A 42 (2) (1990) 789-794. doi:10.1103/PhysRevA.42.789.

[30] G. Galliéro, C. Boned, Thermal conductivity of the Lennard-Jones chain fluid model, Physical Review E 80 (6) (2009) 061202. doi:10.1103/PhysRevE.80.061202. URL https://link.aps.org/doi/10.1103/PhysRevE.80.061202

[31] C. Hoheisel, Memory functions and the calculation of dynamical properties of atomic liquids, Computer Physics Reports 12 (2) (1990) 29 - 66. doi:https://doi.org/ 10.1016/0167-7977(90)90003-0.

URL http://wWw.sciencedirect.com/science/article/pii/0167797790900030

[32] V. G. Baidakov, S. P. Protsenko, Metastable Lennard-Jones fluids. II. Thermal conductivity, The Journal of Chemical Physics 140 (21) (2014) 214506. doi: $10.1063 / 1.4880958$

URL http://aip.scitation.org/doi/10.1063/1.4880958

[33] A. Eskandari Nasrabad, R. Laghaei, B. C. Eu, Molecular theory of thermal conductivity of the Lennard-Jones fluid, The Journal of Chemical Physics 124 (8) (2006) 084506. doi:10.1063/1.2166394.

URL http://aip.scitation.org/doi/10.1063/1.2166394

[34] E. M. Gosling, I. McDonald, K. Singer, On the calculation by molecular dynamics of the shear viscosity of a simple fluid, Molecular Physics 26 (6) (1973) 1475-1484. doi:10.1080/00268977300102631.

URL http://www.tandfonline.com/doi/abs/10.1080/00268977300102631

[35] G. Galliéro, C. Boned, A. Baylaucq, Molecular Dynamics Study of the Lennard-Jones Fluid Viscosity: Application to Real Fluids, Industrial \& Engineering Chemistry 
Research 44 (17) (2005) 6963-6972. doi:10.1021/ie050154t.

URL http://pubs .acs .org/doi/abs/10.1021/ie050154t

[36] D. M. Heyes, Self-diffusion and shear viscosity of simple fluids. A molecular-dynamics study, Journal of the Chemical Society, Faraday Transactions 279 (12) (1983) 1741. doi:10.1039/f29837901741.

URL http://xlink.rsc .org/?DOI=f29837901741

[37] J. Michels, N. Trappeniers, Molecular dynamical calculations of the viscosity of lennard-jones systems, Physica A: Statistical Mechanics and its Applications 133 (1) (1985) 281 - 290. doi:https://doi.org/10.1016/0378-4371(85)90067-6.

URL http://www.sciencedirect.com/science/article/pii/0378437185900676

[38] M. Schoen, C. Hoheisel, The shear viscosity of a Lennard-Jones fluid calculated by equilibrium molecular dynamics, Molecular Physics 56 (3) (1985) 653-672. doi:10.1080/00268978500102591.

$\mathrm{URL}$ http://dx.doi.org/10.1080/00268978500102591\{\%\}5Cnhttp:// www. tandf online.com/doi/abs/10.1080/00268978500102591\{\%\}5Cnhttp: //www.tandfonline.com/doi/abs/10.1080/00268978500102591http://www . tandfonline.com/doi/abs/10.1080/00268978500102591

[39] R. L. Rowley, M. M. Painter, Diffusion and viscosity equations of state for a Lennard-Jones fluid obtained from molecular dynamics simulations, International Journal of Thermophysics 18 (5) (1997) 1109-1121. arXiv:1503.05249v1, doi: 10.1007/BF02575252.

URL http://link . springer .com/10.1007/BF02575252

[40] J. Michels, N. Trappeniers, The self-diffusion coefficient in the gas phase at moderate densities, obtained by computer simulations, Physica A: Statistical Mechanics and its Applications 90 (2) (1978) 179-195. doi:10.1016/0378-4371(78)90108-5.

URL http://linkinghub .elsevier.com/retrieve/pii/0378437178901085

[41] J. E. Straub, Analysis of the role of attractive forces in self-diffusion of a simple fluid, 
Molecular Physics 76 (2) (1992) 373-385. doi:10.1080/00268979200101391. URL http://www . tandfonline.com/doi/abs/10.1080/00268979200101391

[42] V. Baidakov, S. Protsenko, Z. Kozlova, The self-diffusion coefficient in stable and metastable states of the Lennard-Jones fluid, Fluid Phase Equilibria 305 (2) (2011) 106-113. doi:10.1016/j.fluid.2011.03.002.

URL http://dx.doi.org/10.1016/j.fluid.2011.03.002http://linkinghub. elsevier.com/retrieve/pii/S0378381211001257

[43] M. Thol, G. Rutkai, R. Span, J. Vrabec, R. Lustig, Equation of State for the LennardJones Truncated and Shifted Model Fluid, International Journal of Thermophysics 36 (1) (2015) 25-43. doi:10.1007/s10765-014-1764-4.

URL http: //link .springer .com/10.1007/s10765-014-1764-4

[44] P. D. Neufeld, A. R. Janzen, R. A. Aziz, Empirical Equations to Calculate 16 of the Transport Collision Integrals $\Omega(1, \mathrm{~s}$ )* for the Lennard-Jones (12-6) Potential, The Journal of Chemical Physics 57 (3) (1972) 1100-1102. doi:10.1063/1.1678363.

URL http://aip.scitation.org/doi/10.1063/1.1678363

[45] Y. Rosenfeld, Relation between the transport coefficients and the internal entropy of simple systems, Physical Review A 15 (6) (1977) 2545-2549. doi:10.1103/PhysRevA. 15.2545 .

[46] O. Lötgering-Lin, J. Gross, Group Contribution Method for Viscosities Based on Entropy Scaling Using the Perturbed-Chain Polar Statistical Associating Fluid Theory, Industrial and Engineering Chemistry Research 54 (32) (2015) 7942-7952. doi:10.1021/acs.iecr.5b01698.

[47] M. Hopp, J. Gross, Thermal Conductivity of Real Substances from Excess Entropy Scaling Using PCP-SAFT, Industrial and Engineering Chemistry Research 56 (15) (2017) 4527-4538. doi:10.1021/acs.iecr.6b04289 